\title{
Measures of Cortical Plasticity after Transcranial Paired Associative Stimulation Predict Changes in Electroencephalogram Slow-Wave Activity during Subsequent Sleep
}

\author{
Reto Huber, ${ }^{1,2}$ Sara Määttä, ${ }^{1}$ Steve K. Esser, ${ }^{1}$ Simone Sarasso, ${ }^{1}$ Fabio Ferrarelli, ${ }^{1}$ Adam Watson, ${ }^{1}$ Florinda Ferreri, ${ }^{3}$ \\ Michael J. Peterson, ${ }^{1}$ and Giulio Tononi ${ }^{1}$ \\ ${ }^{1}$ Department of Psychiatry, University of Wisconsin-Madison, Madison, Wisconsin 53719, ${ }^{2}$ University Children's Hospital Zurich, CH-8032 Zurich, \\ Switzerland, and ${ }^{3}$ Department of Neurology, University Campus Biomedico, I-00155 Rome, Italy
}

\begin{abstract}
Sleep slow-wave activity (SWA) is thought to reflect sleep need, increasing in proportion to the previous time awake and decreasing during sleep, although the underlying mechanisms are unclear. Recent studies have shown that procedures presumably leading to local plastic changes in the cerebral cortex can lead to local changes in SWA during subsequent sleep. To further investigate the connection between cortical plasticity and sleep SWA, in this study we used a paired associative stimulation (PAS) protocol, in which median nerve stimuli were followed at different intervals $(25$ or $10 \mathrm{~ms}$ ) by transcranial magnetic stimulation (TMS) pulses to the contralateral cortical hand area. As expected, such a protocol led to a sustained increase (long-term potentiation-like) or decrease (long-term depression-like) of cortical excitability as measured by motor evoked potentials. By using a TMS-compatible high-density electroencephalographic (EEG) system, we also found that, in individual subjects, TMS-evoked cortical responses over sensorimotor cortex changed with different interstimulus intervals. Moreover, during subsequent sleep, SWA increased locally in subjects whose TMS-evoked cortical responses had increased after PAS, and decreased in subjects whose cortical responses had decreased. Changes in TMS-evoked cortical EEG response and change in sleep SWA were localized to similar cortical regions and were positively correlated. Together, these results suggest that changes in cortical excitability in opposite directions lead to corresponding changes in local sleep regulation, as reflected by SWA, providing evidence for a tight relationship between cortical plasticity and sleep intensity.
\end{abstract}

Key words: sleep homeostasis; synaptic plasticity; high-density EEG; transcranial magnetic stimulation; slow oscillations; cortical excitability

\section{Introduction}

Increasing evidence indicates that sleep and neural plasticity are linked. Sleep after learning promotes memory consolidation for declarative and nondeclarative tasks (Karni et al., 1994; Gais et al., 2000; Maquet, 2001; Gais and Born, 2004; Walker and Stickgold, 2004; Stickgold, 2005). Sleep deprivation impairs new learning (Yoo et al., 2007) and compromises long-term potentiation (LTP) (Davis et al., 2003; McDermott et al., 2003).

The best characterized marker of sleep intensity is the amount of slow-wave activity (SWA) (EEG power density, 0.75-4.5 Hz) during non-rapid eye movement (NREM) sleep (Borbély and Achermann, 2000), which increases as a function of previous wakefulness and

Received Nov. 5, 2007; revised June 11, 2008; accepted June 12, 2008.

This work was supported by the Swiss Foundation for Fellowships in Biology and Medicine (R.H.), National Institutes of Health Grant 1R01 NS 055 185-01 (G.T.), and a McDonnell Foundation grant (G.T.). We thank Chiara Cirelli for comments on this manuscript and Andy Alexander at the Keck Center for support with EEG and MRI facilities.

Correspondence should be addressed to Giulio Tononi, Department of Psychiatry, University of WisconsinMadison, 6001 Research Park Boulevard, Madison, WI 53719-1176. E-mail: gtononi@wisc.edu.

DOI:10.1523/JNEUROSCI.1636-08.2008

Copyright $\odot 2008$ Society for Neuroscience $\quad$ 0270-6474/08/287911-08\$15.00/0 declines during sleep. However, the mechanisms underlying the increase in SWA with sleep pressure remain unknown. An important clue has come from work showing that sleep SWA can be regulated locally in the cerebral cortex, pointing to a link between SWA regulation and synaptic plasticity. In a high-density (hd)-EEG study, sleep SWA was locally increased after a visuomotor learning task involving right parietal cortex and after transcranial magnetic stimulation (TMS) induced potentiation of primary motor cortex (Huber et al., 2004, 2007). In a third study, subjects underwent arm immobilization during the day, leading to a decrease in motor performance and in somatosensory evoked potentials (SEPs) and motor evoked potentials (MEPs). This was followed by a local decrease of sleep SWA over sensorimotor cortex (Huber et al., 2006). Together, these experiments suggest that sleep SWA is affected by plastic changes in local cortical circuits and, more specifically, that SWA may increase after manipulations that favor synaptic potentiation and decrease after those that promote synaptic depression (Tononi and Cirelli, 2003, 2006).

To test this hypothesis directly, it is important to investigate whether established paradigms for inducing potentiation or depression of cortical circuits yield the predicted changes in sleep 
SWA. To reduce confounding factors, it would be ideal to use very similar paradigms for inducing potentiation and depression and compare the effects within subjects. For this purpose, we decided to employ a paired associative stimulation (PAS) protocol (Classen et al., 2004), which combines peripheral somatosensory stimuli and TMS pulses applied to contralateral sensorimotor cortex to induce plastic changes in motor circuits. The advantage of this paradigm is that, by merely manipulating the interval between the two stimuli by a few milliseconds (typically 10 vs $25 \mathrm{~ms}$ ), one can obtain opposite changes in response strength, as measured by MEPs. The PAS paradigm was developed based on the principles of spike time-dependent plasticity (Abbott and Nelson, 2000), according to which presynaptic activity that precedes postsynaptic firing should induce potentiation, whereas reversing the order should cause depression.

However, the precise effects of the PAS protocol on cortical circuits are mostly unknown, and even at the level of the MEPs there is great variability in the effects produced by different intervals (Fratello et al., 2006). Additional variability is expected at the cortical level because of the fact that TMS is likely to trigger polysynaptic circuits that introduce variable delays and therefore different effects at different synapses. Moreover, in different subjects TMS is likely to affect some circuits and not others (Sakai et al., 1997), so that a PAS interval that produces predominantly potentiation in some areas in one subject may produce depression in other areas and subjects. Because of this variability, a meaningful comparison between changes in circuit strength induced by PAS and the subsequent changes in sleep SWA should be based on the actual effects produced in each individual subject (predominant potentiation or depression) rather than on the interval used. Moreover, because sleep SWA is measured over the entire cortical surface, it is important to establish to what extent overall cortical responses, as opposed to merely motor responses, are modified by different intervals in the PAS protocols.

For this reason, we took advantage of a system that permits hd-EEG recordings of the responses elicited by TMS (Massimini et al., 2005) to directly evaluate changes in the strength of cortical responses after different PAS intervals, and to do so at sufficient spatial resolution to uncover differential effects at different locations. In this way, we were able to directly relate changes in cortical response strength with subsequent changes in sleep SWA.

\section{Materials and Methods}

Design. Nineteen healthy right-handed male subjects (mean age, $25.2 \pm$ 1.0 years) gave informed consent to participate in the study, which was approved by the local ethics committee. We performed a neurological screening to exclude subjects with conditions that could predispose them to potential adverse effects of TMS. The TMS sessions took place in the evening (between 10:00 and 11:00 P.M.). A 60-channel EEG cap was prepared on each subject with scalp impedances $<5 \mathrm{k} \Omega$. The design of the experiment is depicted in Figure 1. Briefly, when the subjects were ready, we collected motor evoked potentials followed by TMS evoked potentials in the pre-PAS period. The $30 \mathrm{~min}$ PAS was then followed by the collection of motor evoked potentials and TMS evoked potentials in the post-PAS period. Equipment adjustments (TMS coil and SEP electrodes placement, etc.) between the different parts of data acquisition resulted in short breaks of a few minutes between the tests. After a 5-10 min break, the room was darkened and subjects were allowed to sleep in a bed while their EEG was recorded. Our EEG electrode system permitted only the acquisition of a first sleep cycle $(1-2 \mathrm{~h})$. The sleep recording was therefore terminated at the first occurrence of REM sleep or when the subject woke up. All reported satisfactory, restful sleep. Subjects underwent three experimental sessions, PAS with an interstimulus interval (ISI) of $10 \mathrm{~ms}$, PAS with an ISI of $25 \mathrm{~ms}$, and sham. Sessions were separated by 1 week and their order was randomized. Data of several subjects were of insufficient quality (because of artifact contamination) to be included in the full analysis. Thus, subject numbers are stated wherever the full number of subjects is not included.

Transcranial magnetic stimulation. The stimulation setup consisted of a Magstim Rapid magnetic stimulator (Magstim) and a figure-of-eight TMS coil (Magstim Double $70 \mathrm{~mm}$ ). Precision and reproducibility of the stimulation were achieved by means of a navigated brain stimulation (NBS) system (Nexstim). The NBS device uses an optical tracking system to locate the TMS coil relative to the subject's coregistered magnetic resonance (MR) image and allows a digitization of the location of the EEG electrodes. T1-weighted MR images (resolution, $0.5 \mathrm{~mm}$ ) of the subjects' whole heads were acquired with a 3T GE Signa scanner. The NBS system delivered trigger pulses that synchronized the TMS, EMG, and EEG systems. To locate the optimal site of TMS, subjects' primary motor cortex was extensively mapped around the anatomically defined "motor knob," the anatomy associated with activation during thumb motion (Denslow et al., 2005), to find the area evoking the largest response in right abductor pollicis brevis (APB muscle), which was chosen as the hot spot. The coil was placed tangentially to the scalp with the handle pointing backwards and $45^{\circ}$ away from the midline. The scalp hot spot varied across subjects, encompassing an area of left central electrodes (numbers $27,28,37,38$ ). Stimulus intensity was set relative to resting motor threshold (RMT), which was determined using a maximum-likelihood threshold hunting procedure and as the TMS intensity producing at least five MEPs $\geq 50 \mu \mathrm{V}$ (peak-to-peak) of 10 consecutive trials (Rossini et al., 1994; Awiszus, 2003). The RMT was $64.2 \pm$ $2 \%$ of the maximal stimulator output. All stimuli were delivered to the same cortical target during the experiment. The electric field induced on the cortex at RMT was estimated at $102.5 \pm 7.7 \mathrm{~V} / \mathrm{m}$.

SEPs. SEPs were evoked by electric stimulation of the median nerve at the right wrist using constant current square-wave pulses $(0.5 \mathrm{~ms})$ with intensity just above the thenar motor threshold.

MEPs. Continuous on-line EMG and MEPs were measured from an electrode pair attached to the skin overlying the APB muscle and the first metacarpophalangeal joint by means of the Nexstim amplifier (see below). We assessed changes in MEP amplitude in a MEP test phase before and after the paired associate stimulation. MEPs were generated by delivering 20 TMS pulses, one every $10 \mathrm{~s}$ at 130\% RMT.

TMS evoked potentials. EEG responses to TMS were recorded by means of a cap with 60 carbon electrodes and a specifically designed TMS-compatible amplifier (Nexstim). TMS was targeted to the hot spot of the APB muscle of the left hemisphere at 90\% RMT. The EEG signals were filtered $(0.1-500 \mathrm{~Hz})$ and sampled at $1450 \mathrm{~Hz}$ (for details, see Massimini et al., 2005). Confounding factors such as auditory evoked responses and attentional effects on evoked responses were reduced by noise masking and by engaging the subject in a simple oddball task. In this task, interspersed within the noise masking, tones were played at irregular intervals $(10-60 \mathrm{~s})$ and the subject had to respond as fast as possible with a mouse button click with the left hand. Differences were assessed by paired $t$ tests. Off-line, for the analysis of evoked responses, the data were average referenced, baseline corrected (100 ms prestimulus), bandpass filtered (5-100 Hz), and averaged for each subject. As in the study by Esser et al. (2006), we used zero padding over the first $15 \mathrm{~ms}$ to remove magnetic artifacts. In addition, in individual subjects, source reconstruction was used to localize the origin of the average electrical activity of each peak of the evoked response. A failure to locate a source within the brain was considered evidence for artifactual contamination. Total EEG activity was assessed using the global mean field power (GMFP) (Lehmann and Skrandies, 1980). TMS evoked potentials were assessed by delivering 200 TMS pulses with an interstimulus interval jittering randomly between 0.5 and $0.7 \mathrm{~s}$ at $90 \%$ RMT.

PAS. PAS consisted of 90 electrical stimuli of the right median nerve at the wrist (see above, SEPs) paired with a single TMS pulse over the motor representation of the right APB muscle of the left hemisphere (hot spot) at 130\% RMT (see above, MEPs) every $15 \mathrm{~s}$. The ISI between peripheral and TMS stimuli was either $10 \mathrm{~ms}$ (ISI 10) or $25 \mathrm{~ms}$ (ISI 25), which was shown previously to induce long-lasting decreases [long-term depression (LTD)-like] or increases (LTP-like) in MEP amplitude, respectively (Stefan et al., 2000, 2002). For safety reasons, the subjects' $E E G$ was carefully monitored on-line during the PAS sessions. We found no epileptiform EEG abnormalities. 


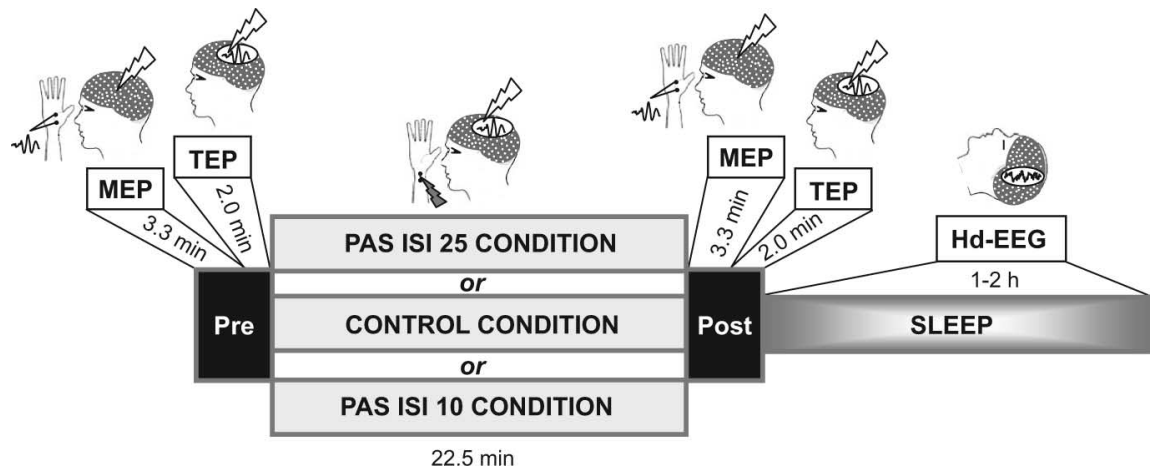

Figure 1. Study design. During the pre and the post test sessions, we collected MEPs followed by TMS-evoked EEG potentials (TEPs). The following three conditions were separated by a week with randomized order: (1) a PAS with an ISI of $25 \mathrm{~ms}$, (2) a PAS with ISI of $10 \mathrm{~ms}$, and (3) a sham control. Subjects were blind to the condition. In the darkened room, the first sleep cycle was recorded using hd-EEG.

A

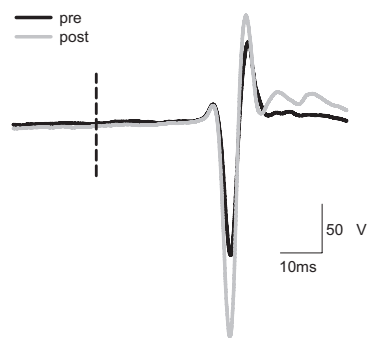

B

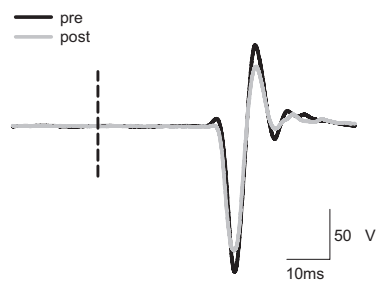

Figure 2. Motor evoked potentials. Responses to TMS before and after paired associate stimulation with an interstimulus interval of $25 \mathrm{~ms}$ (ISI 25) and $10 \mathrm{~ms}$ (ISI 10). A, B, Average of 20 MEPs produced by stimulation at $130 \%$ RMT before (in black) and after PAS (in color). C, Compared with baseline, MEPs were significantly increased in amplitude after PASISI 25 and decreased after PAS ISI $10\left[{ }^{*} p<0.05\right.$, paired $t$ test; $n=15 ; 1$ subject showed a nonsignificant reversed effect (i.e., increased after PAS ISI 10 and decreased after PAS ISI 25); 8 subjects showed a nonsignificant reversed effect either after PAS ISI 25 or after PAS ISI 10]. A direct comparison revealed a significant difference between the two PAS conditions ( $p<0.01$, paired $t$ test). Error bars indicate SE.

Furthermore, subjects were interviewed immediately after and 1 week after the experiment and reported no adverse effects. For sham PAS, the coil was rotated $90^{\circ}$ around the axis of the handle and separated from the head using a $2 \mathrm{~cm}$ plastic spacer cube to ensure an indirect contact between the coil and the head. The right median nerve stimulation was applied as in the experimental conditions. At debriefing at the end of the experiment, subjects did not report any difference among the three conditions.

Sleep recording. Sleep EEG recordings for the first NREM sleep episode were bandpass filtered between 0.1 and $40 \mathrm{~Hz}$, downsampled to $128 \mathrm{~Hz}$ (Matlab function "resample"; Mathworks), and average-referenced. Sleep stages were visually scored in $20 \mathrm{~s}$ epochs according to standard criteria (Rechtschaffen and Kales, 1968). For a quantitative analysis of the sleep EEG, spectral analysis of consecutive 20 s epochs was performed for all 60 channels (fast Fourier transform routine; Hanning window; averages of five $4 \mathrm{~s}$ epochs). Visual and semiautomatic artifact removal was performed (Huber et al., 2000). Significant topographical differences in hd-EEG power during the first 20 min slow-wave sleep (SWS) (NREM sleep stages 2-4) were assessed by statistical nonparametric mapping ( $\mathrm{SnPM})$ using the single threshold test (Nichols and Holmes, 2002; Huber et al., 2004, 2006). This method takes advantage of the actual data distribution and accounts for multiple comparisons testing in hd-EEG recordings. Briefly, EEG readings at each electrode for an experimental condition and the control condition were shuffled according to all possible permutations for all subjects. Based on the statistics obtained from the permutation data, we calculated a $t$ value for each electrode, and found the maximal $t$ value over all electrodes for each permutation. The $t$ value threshold was taken as the 95th percentile of the permutation-derived $t$ values, and electrodes exceeding that threshold were taken as showing a significant difference between the two conditions. The $t$ values presented in the figures are based on paired $t$ tests. For the topograph-

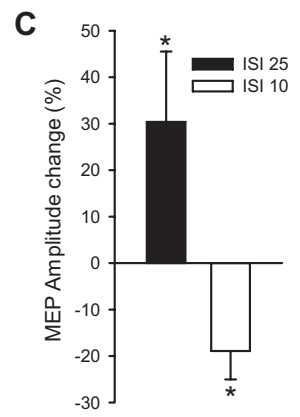

ical display of EEG activity, we used the topoplot function of the EEGLab Matlab toolbox (Delorme and Makeig, 2004). Throughout the paper, variability is represented by SEs.

Source localization. Source localization was performed on the average preconditioning TMS-evoked EEG response using the Curry software package (Curry 5.0; Neuroscan). Electrode positions were digitized and coregistered to each subject's magnetic resonance image (MRI) by means of an infrared positioning system (Nexstim). We then estimated the current density on the cortical surface by using the sLORETA algorithm (Pascual-Marqui, 2002). The current density of the average evoked response was then projected onto the Montreal Neurological Institute (MNI) standard brain.

\section{Results}

The design of the experiment is depicted in Figure 1 (for details, see Materials and Methods). Changes in cortical excitability after PAS were first evaluated using MEP produced by TMS. Changes to cortical response strength were directly evaluated using a dedicated, TMS-compatible EEG system (Massimini et al., 2005). To ensure the precise localization and reproducibility of stimulation sites and the coregistration with EEG responses, we used an optical tracking system based on each subject's magnetic resonance images. In this way, we could (1) use the same paradigm to induce either LTP- or LTD-like changes by merely varying the PAS interval from 10 to $25 \mathrm{~ms}$, (2) compare the effects of LTP-PAS, LTD-PAS, and sham TMS in the same subject, assuring precise reproducibility of the site of stimulation, (3) obtain a topographic map of changes in cortical evoked responses in addition to traditional MEPs, and (4) correlate the changes in TMSevoked EEG responses with local changes in sleep SWA.

\section{TMS response before and after PAS}

We first examined each subject's peripheral and cortical responses to TMS before and after PAS. As expected, we found that motor responses to TMS (measured as the amplitude of MEPs) were significantly increased or decreased after PAS ISI 25 or 10, respectively (Fig. 2 ). However, it is noteworthy that responses varied considerably among individuals, such that some subjects even showed reversed responses (i.e., increased MEP amplitude after ISI 10 and decreased amplitude after ISI 25) (supplemental Fig. S1 A, available at www.jneurosci.org as supplemental material). In a subgroup of subjects, we also recorded SEPs, which showed the classical cortical components with the first negative deflection around $20 \mathrm{~ms}$ (N20) and the largest amplitude between 35 and $45 \mathrm{~ms}(n=10)$.

Next, we analyzed the cortical responses to TMS targeted to the hot spot of the APB muscle of the left hemisphere, directly probing the excitability of the underlying cortex. We first performed source localization of the activity occurring during each peak in the GMFP to visualize which cortical areas were activated by TMS. Figure 3 shows the TMS-induced activity in a single subject before the sham condition (the first two deflections were omitted because of TMS artifacts). TMS produced large deflections in scalp voltage primarily near the site of stimulation but also on the contralateral side. This 
activity lasted for $\sim 200 \mathrm{~ms}$. Very similar spatial activation patterns were obtained after the sham condition as well as after the two active TMS conditions.

We then determined the time course of the total EEG response to TMS by calculating the GMFP for the two experimental conditions across all subjects. Under both conditions, the GMFP contained distinct peaks, which had similar latencies when compared between the pre- and post-TMS test phases. Figure 4 shows the average GMFP difference between the pre and post phase: marked differences can be observed both between each experimental condition and the baseline (blue bars for ISI 10; red bars for ISI 25) and between the two conditions (black bars) (i.e., responses of higher amplitude after PAS ISI 25 and of lower amplitude after ISI 10). There was no response change between pre- and post-TMS test phases for the sham condition.

For a topographical analysis of the TMS-induced responses, we integrated activity in the time ranges at which significant differences between the two conditions occurred for each subject. For all conditions, we observed the strongest evoked activity in electrodes just anterior and posterior to the site of stimulation (Fig. 5). When contrasting the pre and post map of the integrated activity, we found reduction of activity anterior to the stimulation site after the PAS ISI $10(p<$ 0.05, SnPM, for electrode 17) and increased activity spanning the stimulation site after the PAS ISI $25(p<0.05$, SnPM, maxima at electrodes 28, 29, 37, 38, and 49). Furthermore, after PAS ISI 25, several electrodes contralateral to the stimulation site showed significant increases (Fig. 5).

Similar to the observed interindividual differences in changes of the MEP amplitude, the induced change in total EEG activity after PAS was highly variable across subjects (variability, $\pm 18 \%$ after PAS ISI $10, \pm 26 \%$ after PAS ISI 25 , and $\pm 8 \%$ after the sham condition) (supplemental Fig. $\mathrm{S} 1 B$, available at www.jneurosci.org as supplemental material). This is illustrated in Figure $6 A$, in which the change in the integrated GMFP for the time ranges showing significant changes after PAS is depicted for each individual. Activity after PAS shows a tendency for an increase after ISI 25 ( $p<0.1$, paired $t$ test) and a nonsignificant decrease after ISI 10 , however with a large overlap. The activity change after PAS ISI 25 and ISI 10 differed significantly in a direct comparison $(p<0.05$, paired $t$ test). To determine to what extent MEP changes predict the TMS-evoked response, we correlated the MEP amplitude change with the change of the integrated activity of the TMS-evoked response at each electrode across both conditions. We observed significant correlations between the MEP and TMS-evoked response over

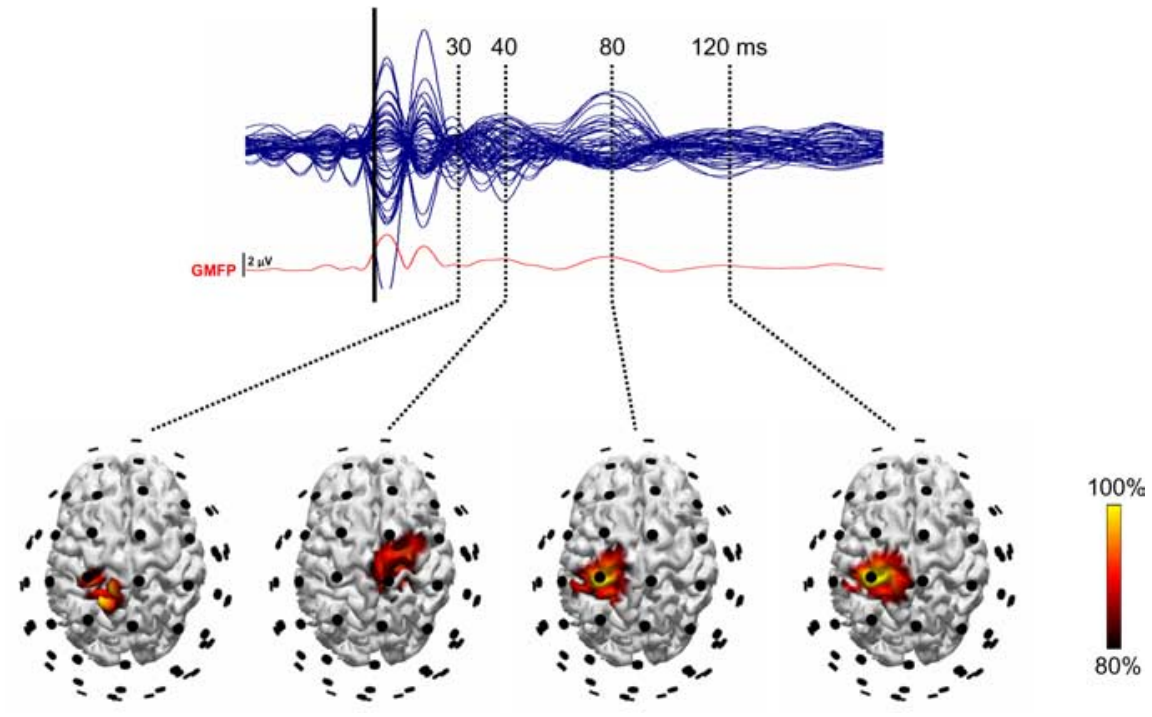

Figure 3. Source localization of TMS-evoked EEG potentials. Top, Averaged TMS evoked potentials recorded at all electrodes, superimposed in a butterfly diagram (black vertical bar represents the time point of TMS application). The red curve below represents the corresponding GMFP. Bottom, Source localization of the activity occurring during each peak in the GMFP (because of TMS artifact contamination the first two peaks were omitted). The top 20\% of current produced is shown.

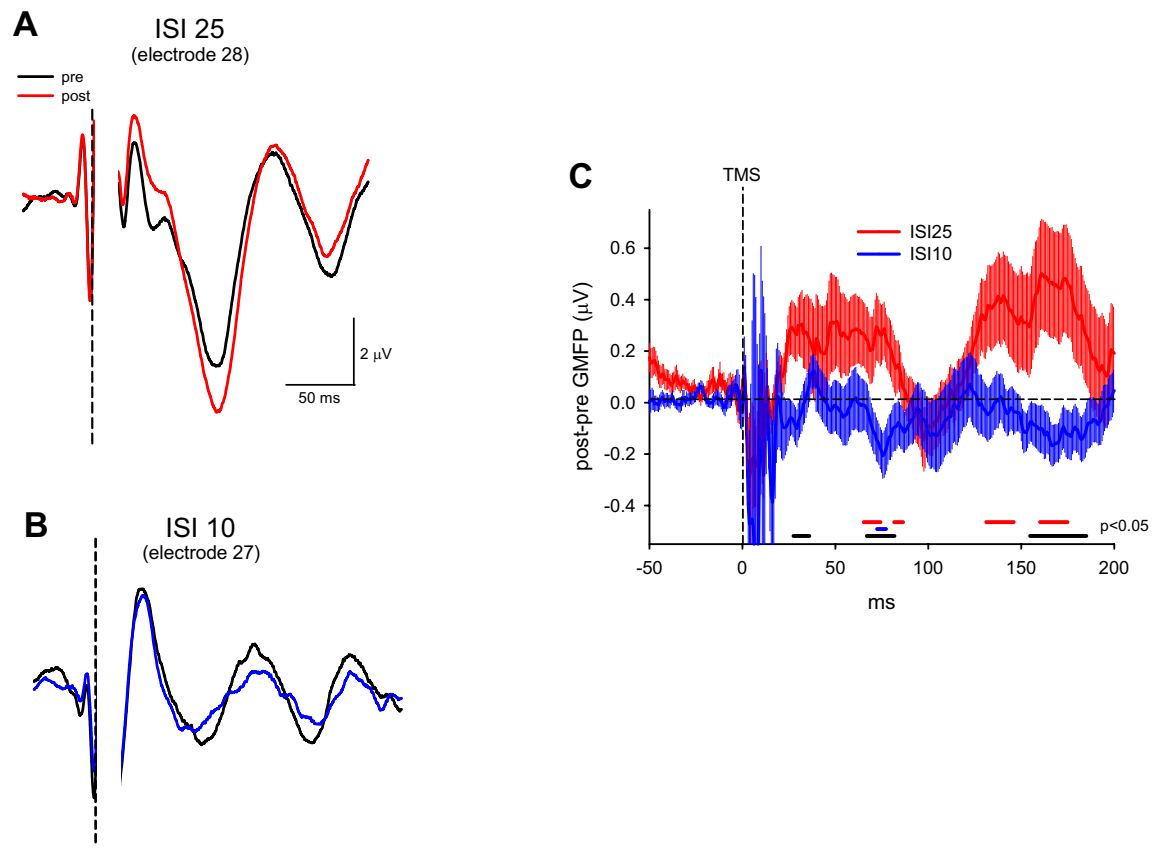

Figure 4. Change in TMS evoked potentials. $A, B$, Average TMS evoked potential at a single electrode for one individual, before (in black) and after (in color) paired associate stimulation with an interstimulus interval of $25 \mathrm{~ms}$ (A) (ISI 25; electrode 28) and 10 ms (B) (ISI 10; electrode 27). C, Average GMFP difference for all subjects between the post and pre test for PASISI 25 (red; $n=16$ ) and ISI 10 (blue; $n=17$ ). The vertical thin lines representSEs. The bottom horizontal lines indicate significant differences between pre and post test (ISI 25, red; ISI 10, blue; $p<0.05$, paired $t$ test) and the significant changes in the direct comparison of the difference between pre and post test for the two conditions in black. left motor cortex $(r=0.52)$, left premotor cortex $(r=0.48)$, and right sensorimotor cortex $(r=0.55)$.

Finally, we addressed the main goal of this study by examining the relationship between potentiation/depression of cortical responses to TMS and subsequent changes in SWA. Therefore, we grouped the subjects for additional analysis according to whether they increased or decreased their EEG response, as measured by a change in GMFP, regardless of the protocol applied. 
A

PAS ISI 25
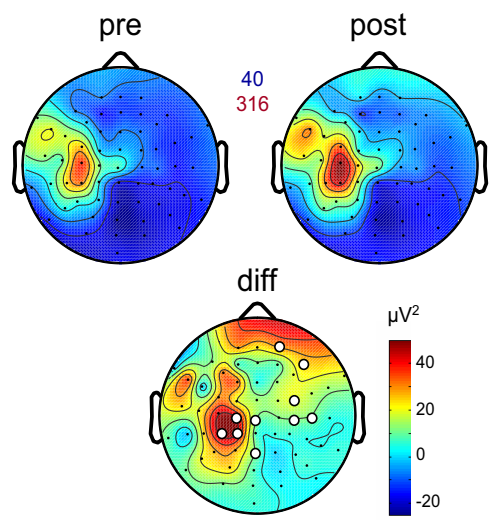

B

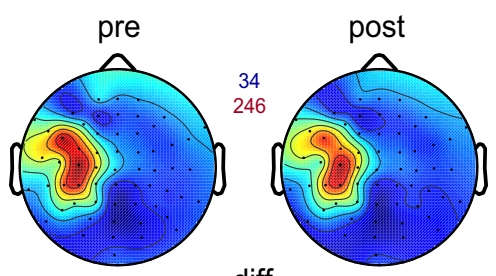

diff

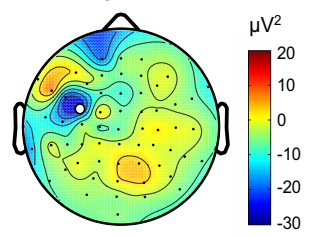

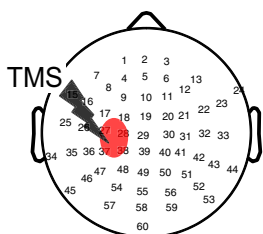

Figure 5. Topographic distribution of the TMS evoked activity. $\boldsymbol{A}, \boldsymbol{B}$, Average integrated evoked response for the time ranges showing significant changes in GMFP between the two conditions (Fig. 4C) before and after paired associate stimulation with an interstimulus interval of $25 \mathrm{~ms}(\boldsymbol{A})($ ISI 25; $n=16)$ and $10 \mathrm{~ms}(\boldsymbol{B})($ ISI 10; $n=17)$. Contrasting the pre and post test topography (diff) revealed increased activity underlying the stimulation site after PAS ISI 25 and decreased activity after PAS ISI 10. The white dots indicate significant differences (for more details, see text). The numbers in between the pre and post topographical plots indicate minimal (blue) and maximal (red) values (in square microvolts) within the two plots.

A

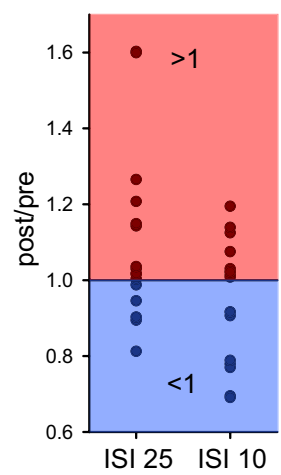

B
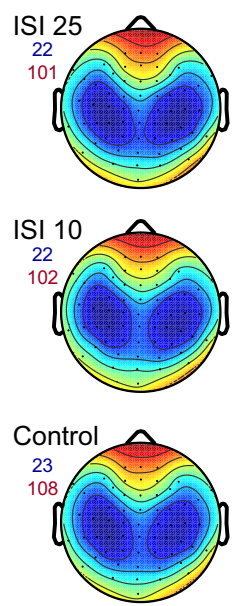

C

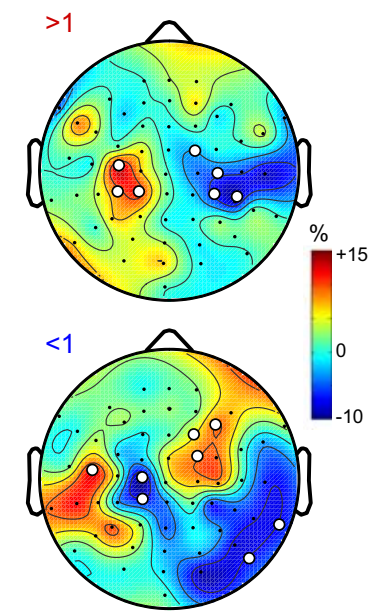

Figure 6. Local SWA changes after PAS. A, Difference of the integrated GMFP for the time ranges showing significant changes in GMFP between the two conditions (Fig. 4C) before and after PAS. All subjects showing increased or decreased total EEG activity, regardless of the condition, were pooled for additional analysis. $\boldsymbol{B}$, Topographic distribution of SWA after paired associate stimulation with an interstimulus interval of $25 \mathrm{~ms}$ (ISI 25) and 10 (ISI 10) and the sham control condition. Average EEG power density at $0.75-4.5 \mathrm{~Hz}$ for the first $20 \mathrm{~min}$ of SWS. Values were normalized by total power for the recording, color coded, plotted at the corresponding position on the planar projection of the scalp surface, and interpolated (biharmonic spline) between electrodes (dots). The numbers below plot labels indicate minimal (blue) and maximal (red) power value (in square microvolts) within each plot. $\boldsymbol{C}$, Topographic distribution of the ratio of SWA between subjects showing increased GMFP according to $\boldsymbol{A}$ (top; $>1 ; n=10$ from $|S| 25$ and $n=8$ from $|S| 10$ condition) or subjects showing decreased GMFP according to $\boldsymbol{A}$ (bottom; $<1 ; n=6$ from $|S| 25$ and $n=9$ from ISI 10 condition) and the control condition. The white dots indicate significant differences.

\section{Sleep SWA after PAS}

After the control and the PAS sessions, the subjects were allowed to sleep and we recorded their sleep EEG. Subjects showed the usual progression of sleep stages in all three conditions, with sleep onset occurring $\sim 20-30 \mathrm{~min}$ after the end of the sessions (sleep latency after lights off: control, $8.9 \pm 1.3$; ISI 25, $6.8 \pm 1.2$; ISI 10, $10.2 \pm 1.8 \mathrm{~min}$; total sleep time: control, $86.9 \pm 5.1$, ISI $25,81.4 \pm$ 4.2. ISI $10,76.8 \pm 5.5 \mathrm{~min}$; percentage wakefulness: control,
$15.5 \pm 3.2$, ISI $25,14.2 \pm 3.5$, ISI 10 , $13.7 \pm 2.9 \%$; percentage NREM sleep stage 1: control, $8.5 \pm 1.6$, ISI $25,7.6 \pm 1.8$, ISI $10,9.2 \pm 2.5 \%$; percentage NREM sleep stage 2: control, $33.3 \pm 3.6$; ISI 25 , $35.1 \pm 3.6$; ISI 10, $35.2 \pm 3.5 \%$; percentage slow-wave sleep: $40.4 \pm 5.0$; ISI 25, $40.3 \pm$ 6.7 ; ISI $10,38.2 \pm 5.5$; percentage movement time: control, $1.4 \pm 0.2$; ISI 25, $1.0 \pm$ 0.2 ; ISI $10,0.8 \pm 0.15 \%)$.

For a visualization of the initial topographic distribution of SWA, for each electrode we calculated the average power in the SWA frequency range during the first $20 \mathrm{~min}$ of SWS (NREM stages 3 and 4, occurring $\sim 40-90$ min after the end of the sessions). In accordance with previous studies (Werth et al., 1997; Finelli et al., 2001), we found that SWA was prevalent in anterior regions and highly reproducible across nights (Fig. 6B). Next, we contrasted the conditions to highlight local differences between the PAS and the control session. During sleep after PAS, the group of subjects (either ISI 25 or ISI 10) showing increased GMFP activity compared with the control condition showed a significant increase of SWA at a cluster of three left central electrodes (Fig. 6C, top) (electrodes $27,37,38$; SnPM, $p<0.05$ ). The same comparison revealed an additional significant decrease of SWA at a cluster of right central electrodes (Fig. 6C, top) (electrodes 20, 31, 41,42 ; SnPM, $p<0.05$ ).

For an anatomical localization of these local changes, all electrodes were digitized and coregistered with the subject's magnetic resonance images. The electrodes showing a significant increase or decrease of SWA were then projected onto the brain. The cluster of electrodes showing increased SWA after PAS was localized to the left sensorimotor cortex (Brodmann areas 1-4). Similarly, the cluster of electrodes with decreased SWA was localized to right sensorimotor cortex (Brodmann areas 1-4).

We also compared the distribution of SWA during sleep in all subjects (either ISI 25 or ISI 10) showing decreased GMFP activity after PAS, and found decreased SWA in a cluster of left central electrodes (Fig. $6 C$, bottom) (electrodes 28, 38; SnPM, $p<$ 0.05 ), which was associated with increased SWA in contralateral electrodes (Fig. 6C, bottom) (electrodes 11, 12, 21; SnPM, $p<$ 0.05 ) and ipsilateral electrodes (electrodes 26; SnPM, $p<0.05)$. Again, the local changes were localized to left and right sensorimotor cortex (Brodmann areas 1-4, 6 for the left decrease; 4, 6 for the right, and 4 and 43 for the left increase).

We also computed SWA topography changes for subgroups of subjects with the same directional change in GMFP activity within each PAS condition. We found similar local changes for subjects showing increased GMFP after PAS with the ISI 25 and the ISI 10 condition. Similarly, subjects showing decreased 


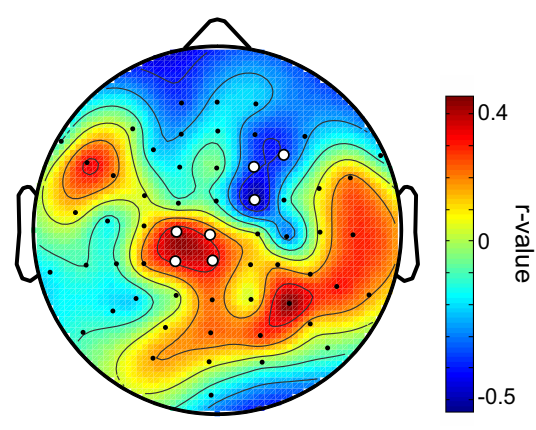

Figure 7. The change in TMS-evoked responses predicts the local change of SWA. Topographic distribution of the correlations between the SWA change and the change in the TMSevoked response after PAS. The change in the integrated global mean field power for the time ranges showing a significant change (Fig. 4C) before and after PAS was correlated with the change in SWA across all subjects at each electrode. The white dots indicate electrodes showing a significant correlation $(p<0.05 ; n=18)$.

GMFP after PAS showed comparable SWA topographies, regardless of whether they were subjected to PAS with ISI 25 or ISI 10 (data not shown).

Next, we asked whether the local changes of SWA were predicted by the changed activity of the TMS-evoked responses induced by PAS. Figure 7 illustrates, topographically across all electrodes, the correlation values between the change in GMFP for the time ranges showing significant differences between the two conditions (Fig. 4C) and the change in SWA during subsequent sleep. We found positive correlations for four electrodes $(27,28$, 37 , and 38) overlying the site of stimulation. Negative correlations were found for contralateral electrodes (11, 12, and 20).

Homeostatic changes in sleep pressure as a function of previous wakefulness are reflected in the global sleep EEG power spectrum mainly as changes in SWA (Borbély and Achermann, 2000). To examine whether the local EEG changes after PAS shared key features with the global homeostatic response observed in the sleep EEG we examined, for each frequency bin, whether there was a correlation between the PAS-induced change in EEG power and the PAS-related change in GMFP. Consistent with a homeostatic response, we found that significant correlations occurred exclusively in the low SWA frequency range (supplemental Fig. S2, available at www.jneurosci.org as supplemental material). No significant correlations were found when the change in sleep EEG power was correlated with PAS-related change in MEP amplitude (data not shown).

Because of technical limitations (see Materials and Methods), our sleep analysis was restricted to the first NREM sleep episode. We found lasting effects in the sleep EEG up to 90 min after the PAS session. Future studies will be required to show how this effect evolves in the course of a night.

In summary, PAS caused local changes in the amplitude of EEG responses to TMS pulses, indicative of changes in the responsiveness of sensorimotor circuits. These changes were followed, during subsequent sleep, by corresponding local changes in SWA: increased SWA was found in subjects whose cortical responsiveness had increased, and decreased SWA in subjects whose cortical responsiveness had decreased after PAS. Moreover, the change of the EEG response to TMS pulses and the change in sleep SWA were localized to a similar cortical region and were positively correlated.

\section{Discussion}

\section{PAS and cortical excitability}

In vitro studies have shown that presynaptic activity that precedes postsynaptic firing or depolarization by a few tens of milliseconds can induce LTP, whereas reversing this temporal order causes LTD (Levy and Steward, 1983; Gustafsson et al., 1987; Debanne et al., 1994). Spike timing-dependent plasticity is a key mechanism governing moment-to-moment changes in synaptic efficacy in cortical circuits (Magee and Johnston, 1997; Markram et al., 1997; Karmarkar et al., 2002; Watanabe et al., 2002), which appears to depend on NMDA receptor activation (Linden, 1999; Karmarkar et al., 2002; Watanabe et al., 2002).

The PAS protocol was devised with the explicit purpose of exploiting the mechanisms of spike timing-dependent plasticity to induce lasting changes in cortical excitability (Classen et al., 2004). In practice, PAS employs median nerve stimulation in conjunction with TMS of contralateral sensorimotor cortex at different ISIs to induce lasting changes in cortical excitability (up to $1 \mathrm{~h}$ ), which are demonstrated by comparing TMS-induced MEPs before and after PAS (Stefan et al., 2000). Most likely, PAS protocols have complicated effects in sensorimotor cortex and in connected areas. Indeed, the data showed high interindividual variability, but on average the effects of PAS on MEPs reverse sign as predicted by spike timingdependent plasticity. Moreover, antagonizing NMDA receptors blocks the increase in MEP amplitude triggered by PAS, whereas intracortical inhibition is unaffected (Stefan et al., 2002). These findings suggest that the main effects of PAS on cortical excitability are likely attributable to LTP-like (or LTD-like) mechanisms and not to changes in the balance between inhibition and excitation (Stefan et al., 2002). However, whether the observed changes in cortical excitability are induced by spike timing-dependent plasticity or other LTP/LTD like mechanisms [e.g., associative cortical afferent LTP (Humeau et al., 2003)] cannot be determined with our experimental setup.

Here, we confirmed that a $25 \mathrm{~ms}$ ISI PAS produces an average increase in MEPs, whereas with a $10 \mathrm{~ms}$ ISI average MEPs are reduced. As noted by others (Fratello et al., 2006), however, the effects of PAS on MEP showed great interindividual variability: we found that in some subjects a $25 \mathrm{~ms}$ delay could depress MEP responses and a $10 \mathrm{~ms}$ delay could potentiate them. Most important, unlike previous studies, we also recorded TMS-evoked cortical responses by combining TMS with simultaneous hd-EEG. Our results provide the first direct evidence that PAS protocols cause changes in TMS-evoked cortical EEG responses, presumably because of underlying changes in the excitability of cortical circuits. PAS-induced potentiation or depression of TMS-evoked EEG responses also showed considerable interindividual variability with respect to ISI, as would be expected given the complex interactions between the subset of stimulated fibers, their conduction delays, and the resulting timing at affected synapses. Moreover, PAS protocols had differential effects on cortical excitability at different locations. Although we found a positive correlation between MEP and change in TMS-evoked responses, this correlation was weak, suggesting that MEPs cannot be considered as an unambiguous indicator of overall changes in cortical excitability.

By analyzing hd-EEG maps of TMS-evoked responses, we found that the most significant changes in cortical excitability after PAS occur near the site of the TMS application over sensorimotor cortex. This site of maximal plasticity overlaid precisely the cortical region in which somatosensory evoked potentials induced by median nerve stimulation overlapped with TMSevoked EEG responses induced by TMS pulses to the motor hot spot (supplemental Fig. S3, available at www.jneurosci.org as supplemental material), thereby supporting the rationale behind the PAS paradigm. In line with our findings, a recent PAS study targeted to the somatosensory cortex localized changes in so- 
matosensory evoked potentials to the area underlying the coil, and showed that these changes were correlated with changes in tactile discrimination (Litvak et al., 2007). A recent study using 5 $\mathrm{Hz}$ repetitive TMS conditioning over motor cortex also found maximal changes in TMS-evoked EEG responses near the site of stimulation (Esser et al., 2006).

hd-EEG recordings also showed that the effects of PAS were not confined to the sensorimotor regions underlying the TMS coil, but extended to the contralateral sensorimotor cortex, although with the opposite sign. A possible explanation for this finding is that the arrival of somatosensory volleys triggered by median nerve stimulation and that of volleys triggered, directly or indirectly, by TMS, reach different brain regions at different time intervals, so that in some areas the predominant effect may be potentiation, and in other areas it may be depression. Consistent with this interpretation, source localization showed that singlepulse TMS targeted to the motor hot spot leads to an initial activation of the ipsilateral primary motor cortex, and next the activation spreads to the contralateral motor cortex and eventually returns to the ipsilateral motor cortex (Fig. 3). Secondary activation of connected cortical areas has been demonstrated by combined TMS-positron emission tomography and TMS-functional magnetic resonance imaging (fMRI) studies (Fox et al., 1997; Bestmann et al., 2003; Ferrarelli et al., 2004). Transcallosal inhibitory connections may also play a role (Ferbert et al., 1992), as suggested by fMRI studies in which unilateral hand movements were associated with contralateral activation and ipsilateral deactivation (Allison et al., 2000).

\section{PAS effects on sleep SWA}

Because the goal of this study was to examine whether plastic changes induced by PAS, as reflected by cortical TMS-evoked responses, are reflected in local changes of sleep regulation, we focused on the relationship between changes in GMFP and SWA, the best characterized marker of sleep homeostasis. It was previously shown that learning a visuomotor task involving right parietal cortex led to a local increase in SWA during the first sleep cycle (Huber et al., 2004). Importantly, this increase was correlated with improvement in performance after sleep. Another recent study showed that boosting slow oscillations by transcranial application of oscillating potentials improves the retention of hippocampus-dependent declarative memories (Marshall et al., 2006). In contrast, arm immobilization, which leads to a deterioration in motor performance and to a decrease in somatosensory responses and MEPs, was followed by a local decrease in sleep SWA over right sensorimotor cortex (Huber et al., 2006). These experiments suggest that SWA may increase after manipulations that favor synaptic potentiation and decrease after those that promote synaptic depression (Tononi and Cirelli, 2003, 2006). However, none of these paradigms could test directly, in the same subjects, whether opposite manipulations of cortical plasticity during wakefulness would lead to corresponding changes in SWA during sleep.

In this study, regional changes in cortical excitability triggered by PAS at different ISIs were compared with subsequent changes in sleep SWA within the same subject. Our experimental design allowed for grouping subjects on the basis of actual changes observed in TMS-evoked EEG responses (potentiation or depression) rather than on the basis of MEP changes or of ISIs (25 or 10 $\mathrm{ms})$. By considering group averages, we found that when PAS produced an increase in cortical excitability during wakefulness, as indicated by an increased amplitude of TMS-evoked EEG responses, it was followed by a local relative increase in sleep SWA.
In contrast, when PAS produced a decrease in cortical excitability during wakefulness, as indicated by reduced TMS-evoked EEG responses, it was followed by a local relative decrease in sleep SWA. Thus, plastic changes in TMS-triggered responses induced by PAS protocols and SWA changes during subsequent sleep had the same sign. Notably, the only difference between the two PAS protocols is a $15 \mathrm{~ms}$ shift in the pairing between somatosensory and TMS stimuli (for a total of just 90 pairings). Thus, it is unlikely that the observed differential modulation of sleep SWA could result from aspecific effects of TMS or somatosensory stimulation, such as differences in the extent of neuronal activation ("use"), the depletion of energy resources, or harmful consequences on the stimulated tissue, because the number of TMS pulses and somatosensory stimuli received by each subject was the same in both sessions. Instead, it is likely that the differential effects are attributable to differential plastic changes induced in local cortical circuits.

The present results also show that changes in the amplitude of TMS-evoked EEG responses during wakefulness were positively correlated with changes in local SWA during subsequent sleep. This positive correlation was significant in the low SWA frequency range $(0.75-1.5 \mathrm{~Hz})$ (supplemental Fig. S2, available at www.jneurosci.org as supplemental material) encompassing the cortical slow oscillations, which are most prominent during SWS (Steriade et al., 1993). Furthermore, the spatial location of changes in cortical excitability corresponded with that of changes in sleep SWA. Depending on the PAS interval, the amplitude of TMS-evoked cortical responses increased or decreased over the stimulated sensorimotor cortex compared with the sham condition, and behaved oppositely on the unstimulated sensorimotor cortex. Sleep SWA followed the same pattern, showing a positive correlation with TMS-evoked cortical responses over the stimulated cortex and a negative correlation over the unstimulated cortex (Fig. 7).

\section{Possible mechanisms linking neuronal plasticity and SWA}

How changes in synaptic efficacy can produce changes in sleep SWA have yet to be explored experimentally. However, largescale computer simulations of SWS in thalamocortical circuits demonstrate that an increase in the strength of excitatory corticocortical connections is sufficient to produce a marked increase in sleep SWA and vice versa (Esser et al., 2007). The simulations also suggest that the effects of synaptic strength on SWA and other slow-wave parameters are mediated by changes in the amplitude of single-cell oscillations attributable to increased EPSP size, in the dynamics of network synchronization attributable to increased neuronal coupling, and in the rate of neuronal recruitment and decruitment. Nevertheless, the local regulation of sleep SWA is compatible with other mechanisms. For example, usedependent changes in the efficacy of inhibitory circuits, accumulation or depletion of substances altering neuronal excitability, or alterations of intrinsic excitability may have similar consequences for the generation and synchronization of sleep slow waves or may at least contribute to the observed effects.

\section{References}

Abbott LF, Nelson SB (2000) Synaptic plasticity: taming the beast. Nat Neurosci 3 [Suppl]:1178-1183.

Allison JD, Meador KJ, Loring DW, Figueroa RE, Wright JC (2000) Functional MRI cerebral activation and deactivation during finger movement. Neurology 54:135-142.

Awiszus F (2003) TMS and threshold hunting. Suppl Clin Neurophysiol 56:13-23.

Bestmann S, Baudewig J, Siebner HR, Rothwell JC, Frahm J (2003) Sub- 
threshold high-frequency TMS of human primary motor cortex modulates interconnected frontal motor areas as detected by interleaved fMRITMS. Neuroimage 20:1685-1696.

Borbély AA, Achermann P (2000) Homeostasis of human sleep and models of sleep regulation. In: Principles and practice of sleep medicine (Kryger MH, Roth T, Dement WC, eds), pp 377-390. Philadelphia: Saunders.

Classen J, Wolters A, Stefan K, Wycislo M, Sandbrink F, Schmidt A, Kunesch E (2004) Paired associative stimulation. Suppl Clin Neurophysiol 57:563-569.

Davis CJ, Harding JW, Wright JW (2003) REM sleep deprivation-induced deficits in the latency-to-peak induction and maintenance of long-term potentiation within the CAl region of the hippocampus. Brain Res 973:293-297.

Debanne D, Gähwiler BH, Thompson SM (1994) Asynchronous pre- and postsynaptic activity induces associative long-term depression in area CA1 of the rat hippocampus in vitro. Proc Natl Acad Sci US A 91:1148-1152.

Delorme A, Makeig S (2004) EEGLAB: an open source toolbox for analysis of single-trial EEG dynamics including independent component analysis. J Neurosci Methods 134:9-21.

Denslow S, Bohning DE, Bohning PA, Lomarev MP, George MS (2005) An increased precision comparison of TMS-induced motor cortex BOLD fMRI response for image-guided versus function-guided coil placement. Cogn Behav Neurol 18:119-126.

Esser SK, Huber R, Massimini M, Peterson MJ, Ferrarelli F, Tononi G (2006) A direct demonstration of cortical LTP in humans: a combined TMS/EEG study. Brain Res Bull 69:86-94.

Esser SK, Hill SL, Tononi G (2007) Sleep homeostasis and cortical synchronization: I. Modeling the effects of synaptic strength on sleep slow waves. Sleep 30:1617-1630.

Ferbert A, Priori A, Rothwell JC, Day BL, Colebatch JG, Marsden CD (1992) Interhemispheric inhibition of the human motor cortex. J Physiol 453:525-546.

Ferrarelli F, Haraldsson HM, Barnhart TE, Roberts AD, Oakes TR, Massimini M, Stone CK, Kalin NH, Tononi G (2004) A [ $\left.{ }^{17} \mathrm{~F}\right]$-fluoromethane PET/ TMS study of effective connectivity. Brain Res Bull 64:103-113.

Finelli LA, Borbély AA, Achermann P (2001) Functional topography of the human nonREM sleep electroencephalogram. Eur J Neurosci 13:2282-2290.

Fox P, Ingham R, George MS, Mayberg H, Ingham J, Roby J, Martin C, Jerabek P (1997) Imaging human intra-cerebral connectivity by PET during TMS. Neuroreport 8:2787-2791.

Fratello F, Veniero D, Curcio G, Ferrara M, Marzano C, Moroni F, Pellicciari MC, Bertini M, Rossini PM, De Gennaro L (2006) Modulation of corticospinal excitability by paired associative stimulation: reproducibility of effects and intraindividual reliability. Clin Neurophysiol 117:2667-2674.

Gais S, Born J (2004) Declarative memory consolidation: mechanisms acting during human sleep. Learn Mem 11:679-685.

Gais S, Plihal W, Wagner U, Born J (2000) Early sleep triggers memory for early visual discrimination skills. Nat Neurosci 3:1335-1339.

Gustafsson B, Wigström H, Abraham WC, Huang YY (1987) Long-term potentiation in the hippocampus using depolarizing current pulses as the conditioning stimulus to single volley synaptic potentials. J Neurosci 7:774-780.

Huber R, Graf T, Cote KA, Wittmann L, Gallmann E, Matter D, Schuderer J, Kuster N, Borbély AA, Achermann P (2000) Exposure to pulsed highfrequency electromagnetic field during waking affects human sleep EEG. Neuroreport 11:3321-3325.

Huber R, Ghilardi MF, Massimini M, Tononi G (2004) Local sleep and learning. Nature 430:78-81.

Huber R, Ghilardi MF, Massimini M, Ferrarelli F, Riedner BA, Peterson MJ, Tononi G (2006) Arm immobilization causes cortical plastic changes and locally decreases sleep slow wave activity. Nat Neurosci 9:1169-1176.

Huber R, Esser SK, Ferrarelli F, Massimini M, Peterson MJ, Tononi G (2007) TMS-induced cortical potentiation during wakefulness locally increases slow wave activity during sleep. PLoS ONE 2:e276.

Humeau Y, Shaban H, Bissiere S, Luthi A (2003) Presynaptic induction of heterosynaptic associative plasticity in the mammalian brain. Nature 426 : 841-845.

Karmarkar UR, Najarian MT, Buonomano DV (2002) Mechanisms and significance of spike-timing dependent plasticity. Biol Cybern 87:373-382.

Karni A, Tanne D, Rubenstein BS, Askenasy JJ, Sagi D (1994) Dependence on REM sleep of overnight improvement of a perceptual skill. Science 265:679-682.

Lehmann D, Skrandies W (1980) Reference-free identification of components of checkerboard-evoked multichannel potential fields. Electroencephalogr Clin Neurophysiol 48:609-621.

Levy WB, Steward O (1983) Temporal contiguity requirements for longterm associative potentiation/depression in the hippocampus. Neuroscience 8:791-797.

Linden DJ (1999) The return of the spike: postsynaptic action potentials and the induction of LTP and LTD. Neuron 22:661-666.

Litvak V, Zeller D, Oostenveld R, Maris E, Cohen A, Schramm A, Gentner R, Zaaroor M, Pratt H, Classen J (2007) LTP-like changes induced by paired associative stimulation of the primary somatosensory cortex in humans: source analysis and associated changes in behaviour. Eur J Neurosci 25:2862-2874.

Magee JC, Johnston D (1997) A synaptically controlled, associative signal for Hebbian plasticity in hippocampal neurons. Science 275:209-213.

Maquet P (2001) The role of sleep in learning and memory. Science 294:1048-1052.

Markram H, Lübke J, Frotscher M, Sakmann B (1997) Regulation of synaptic efficacy by coincidence of postsynaptic APs and EPSPs. Science 275:213-215.

Marshall L, Helgadóttir H, Mölle M, Born J (2006) Boosting slow oscillations during sleep potentiates memory. Nature 444:610-613.

Massimini M, Ferrarelli F, Huber R, Esser SK, Singh H, Tononi G (2005) Breakdown of cortical effective connectivity during sleep. Science 309:2228-2232.

McDermott CM, LaHoste GJ, Chen C, Musto A, Bazan NG, Magee JC (2003) Sleep deprivation causes behavioral, synaptic, and membrane excitability alterations in hippocampal neurons. J Neurosci 23:9687-9695.

Nichols TE, Holmes AP (2002) Nonparametric permutation tests for functional neuroimaging: a primer with examples. Hum Brain Mapp 15:1-25.

Pascual-Marqui RD (2002) Standardized low-resolution brain electromagnetic tomography (sLORETA): technical details. Methods Find Exp Clin Pharmacol 24 [Suppl D]:5-12.

Rechtschaffen A, Kales A (1968) A manual of standardized terminology, techniques and scoring system for sleep stages of human subjects. Bethesda, MD: National Institutes of Health.

Rossini PM, Barker AT, Berardelli A, Caramia MD, Caruso G, Cracco RQ, Dimitrijević MR, Hallett M, Katayama Y, Lücking CH (1994) Noninvasive electrical and magnetic stimulation of the brain, spinal cord and roots: basic principles and procedures for routine clinical application. Report of an IFCN committee. Electroencephalogr Clin Neurophysiol 91:79-92.

Sakai K, Ugawa Y, Terao Y, Hanajima R, Furubayashi T, Kanazawa I (1997) Preferential activation of different I waves by transcranial magnetic stimulation with a figure-of-eight-shaped coil. Exp Brain Res 113:24-32.

Stefan K, Kunesch E, Cohen LG, Benecke R, Classen J (2000) Induction of plasticity in the human motor cortex by paired associative stimulation. Brain 123:572-584.

Stefan K, Kunesch E, Benecke R, Cohen LG, Classen J (2002) Mechanisms of enhancement of human motor cortex excitability induced by interventional paired associative stimulation. J Physiol 543:699-708.

Steriade M, McCormick DA, Sejnowski TJ (1993) Thalamocortical oscillations in the sleeping and aroused brain. Science 262:679-685.

Stickgold R (2005) Sleep-dependent memory consolidation. Nature 437:1272-1278

Tononi G, Cirelli C (2003) Sleep and synaptic homeostasis: a hypothesis. Brain Res Bull 62:143-150.

Tononi G, Cirelli C (2006) Sleep function and synaptic homeostasis. Sleep Med Rev 10:49-62.

Walker MP, Stickgold R (2004) Sleep-dependent learning and memory consolidation. Neuron 44:121-133.

Watanabe S, Hoffman DA, Migliore M, Johnston D (2002) Dendritic K ${ }^{+}$ channels contribute to spike-timing dependent long-term potentiation in hippocampal pyramidal neurons. Proc Natl Acad Sci USA 99:8366-8371.

Werth E, Achermann P, Borbély AA (1997) Fronto-occipital EEG power gradients in human sleep. J Sleep Res 6:102-112.

Yoo SS, Hu PT, Gujar N, Jolesz FA, Walker MP (2007) A deficit in the ability to form new human memories without sleep. Nat Neurosci 10:385-392. 